\title{
Aspect Ratio Effect on Convective Heat Transfer of Radially Outward Flow in Rotating Rectangular Ducts
}

\author{
C. R. KUO and G. J. HWANG \\ Department of Power Mechanical Engineering, National Tsing Hua University, Hsinchu, Taiwan, R.O.C.
}

\begin{abstract}
Experiments were conducted to investigate the effects of rotation and aspect ratio on the convective heat transfer of radially outward air flows in rotating rectangular ducts with a uniform wall heat flux by using fiberglass duct walls lined with separated film heaters. The duct hydraulic diameter, heater active length, and mean rotation radius were 4,120 , and $180 \mathrm{~mm}$, respectively. Ranges of parameters were through-flow Reynolds number, 1,000-15,000; rotation number, $0-0.32$; rotational buoyancy parameter, $0-1.2$; and cross-sectional aspect ratio, $0.5,1.0$, and 2.0. The results showed that the higher the rotation number, the greater the enhancement of the heat transfer rate especially at the pressure side. The rotational buoyancy parameter decreases the heat transfer for low Re but enhances the heat transfer for high Re. The largest heat transfer enhancement is seen for $\mathrm{AR}=1.0$, and the enhancement for $\mathrm{AR}=0.5$ is greater than that for $\mathrm{AR}=2.0$.
\end{abstract}

Key Words: Aspect ratio, convective heat transfer, rotating duct, Coriolis force, centrifugal-buoyancy force, radially outward flow

$\mathbf{T}$ HE study of internal convective heat transfer in rotating ducts is becoming of great significance for engineers because of its potential applications in industry: e.g., cooling of turbine blades and cooling of electrical machinery. Increasing the turbine entry temperature is especially required to improve the thermodynamic efficiency and to reduce the specific fuel consumption for the compact design of advanced gas turbine engines. Increasing the power output of electrical machinery is via the increases in the electrical and magnetic loadings in the stator and rotor of machine. Moreover, high operating temperature might cause material degradation on rotating components and excess ohm loss in electrical conductors; thus efficient internal convective cooling technology introduced by flows in radially rotating duct is increasingly important. In a radially rotating heated rectangular duct the flow structure and the heat transfer mechanism are simultaneously influenced by the rotation and the duct geometry. Many investigations on the effects of rotation and duct geometry on flow and internal heat transfer have been reported over these years.

By obtaining an approximate series solution from a perturbation equation in a rotating pipe flow, Barua [1955] showed that two counter-rotating vortices induced by Coriolis acceleration appear symmetrically in the duct. Mori et al. [1968] studied the laminar convective heat transfer in radially rotating circular ducts by assuming velocity and temperature boundary layer profiles along the pipe wall. Subsequently, by using the same techniques, Mori et al. [1971] analyzed the turbulent convective heat transfer in a circular pipe. Table I lists recent experimental investigations on the internal convective heat transfer in radially rotating ducts. Under uniform wall temperature conditions, Wagner et al. [1991a, 1991b] investigated the local heat transfer of radially outward and inward flows in rotating serpentine passages with smooth walls. Buoyant flow is found to be favourable for heat transfer for both pressure and suction sides. However, the increase in heat transfer for the inward-flowing passage was relatively less than that for outward flow. Morris and GhavamiNasr [1991] observed that centrifugal buoyancy is shown to influence the heat transfer response in a rectangularsectioned duct. Heat transfer is improved on pressure and suction sides as the wall-to-coolant temperature difference is increased for radially rotating outward flows. Han and Zhang [1992] reported the local heat transfer coefficient 
TABLE I

Experimental investigations on heat transfer in radially rotating ducts

\begin{tabular}{|c|c|c|c|c|c|c|c|c|c|}
\hline Author & Year & $\begin{array}{l}\text { Duct } \\
\text { Type }\end{array}$ & $\begin{array}{c}D_{h} \\
(\mathrm{~mm})\end{array}$ & $\begin{array}{l}\text { Max. } \\
\text { rpm }\end{array}$ & $L / D_{h}$ & $R / D_{h}$ & $\operatorname{Re} \cdot 10^{-3}$ & $\begin{array}{c}\text { Max. } \\
\text { Ro }\end{array}$ & B.C. \\
\hline Clifford et al. & 1984 & $\Delta$ & 7.67 & 1,000 & 20 & 33 & $6.8-38.0$ & 0.004 & UHF \\
\hline \multirow[t]{2}{*}{ Harasgama and Morris } & 1988 & $\begin{array}{c}\Delta \\
\square\end{array}$ & $\begin{array}{l}7.67 \\
7.5\end{array}$ & $\begin{array}{l}1,850 \\
1,000\end{array}$ & $\begin{array}{l}20 \\
20\end{array}$ & $\begin{array}{l}33 \\
33\end{array}$ & $\begin{array}{l}7.0-25.0 \\
7.0-21.0\end{array}$ & $\begin{array}{l}0.103 \\
0.042\end{array}$ & $\begin{array}{l}\text { UHF } \\
\text { UHF }\end{array}$ \\
\hline & & 0 & 5.0 & 2,000 & 20 & 70 & $7.0-25.0$ & 0.049 & UHF \\
\hline Guidez & 1989 & $\square$ & 10.66 & 5,000 & 11.5 & 27 & $17.0-41.0$ & 0.2 & UHF \\
\hline Hwang and Soong & 1989 & $\square$ & 4.0 & 3,000 & 30 & 30 & $0.7-20.0$ & 0.428 & UWT \\
\hline Soong et al. & 1991 & Lال & 4.0 & 3,000 & 30 & 30 & $0.7-20.0$ & 0.428 & UWT \\
\hline Morris and Ghavami-Nasr & 1991 & $\square$ & 7.3 & 1,800 & 20 & 34 & $10.0-25.0$ & 0.02 & UHF \\
\hline Wagner et al. $(\mathrm{a}, \mathrm{b})$ & 1991 & $\square$ & 12.7 & 1,100 & 14 & 33,49 & $12.5-50.0$ & 0.48 & UWT \\
\hline Han and Zhang & 1992 & $\square$ & 12.7 & 800 & 12 & 30 & $2.5-25.0$ & 0.352 & UWT, UHF \\
\hline Hwang and Kuo & 1993 & $\square$ & 4.0 & 3,000 & 30 & 45 & $1.0-15.5$ & 0.32 & UHF \\
\hline Present study & 1994 & $\square \square$ & 4.0 & 3,000 & 30 & 45 & $1.0-15.5$ & 0.32 & UHF \\
\hline
\end{tabular}

in a square channel with smooth walls and radial outward flow for cases of uneven wall temperature with experiments. Hwang and Kuo [1993] conducted experiments on radially outward flows in a rotating square duct with uniform wall heat flux. Augmentation of heat transfer on the pressure side is clearly observed.

To increase the effects of rotation on internal heat transfer in the rotating ducts, lightweight and high-strength test sections were built for the requirement of high rotational speed up to $3,000 \mathrm{rpm}$. The interior wall surfaces of duct section were lined with separated stainless-steel film heaters of $0.01-\mathrm{mm}$ thickness for the uniform wall heat flux. The purpose of his study was to investigate the effects of forced flow, rotation, and aspect ration on the convective heat transfer of radially outward flows in heated rotating rectangular ducts. The aspect ratio used were 0.5 , 1.0, and 2.0. Regional average Nusselt numbers on the pressure side, the suction side, and the side walls were obtained.

\section{GOVERNING PARAMETERS}

The physical model and coordinates, as shown in Fig. 1, present the rotation-induced inertial effects on forced convection of radially outward air flow in a rotating rectangular duct. Observing the force diagram, one discerns that the dominant forces due to rotation are force vectors of $2 \rho \Omega U$ and $\rho \Omega^{2} X$ because of $U \gg V$ and $X \gg Y$ and $Z$. The Coriolis force term $(2 \rho \Omega U)$ induces cross streams which create additional mixing to the flow. The centrifugal force term $\left(\rho \Omega^{2} X\right)$ generates centrifugal-buoyant radial secondary flow. The direction of this free convection flow is opposite to that of the radially outward flow in a heated rotating duct. An analysis of the flow-governing equations gives that the heat transfer coefficient at a certain axial location in the heated region is functionally influenced by other operating parameters. The results are (Soong et al. [1991]):

$$
\mathrm{Nu}_{\Omega}=f\left(\operatorname{Re}, \operatorname{Re}_{\Omega}, \operatorname{Ra}_{\Omega}, \operatorname{Pr}, L / D_{h}, R / D_{h}, a / b\right)
$$

The definitions of these dimensionless parameters are listed in the nomenclature.

In the present study, air with $\operatorname{Pr}=0.72$ is used as the coolant fluid. The ratio of heated length and hydraulic diameter $L / D_{h}=30.0$ and the ratio of mean rotation radius and hydraulic diameter $R / D_{h}=45.0$ are also fixed. Thus, Eq. [1] reduces to

$$
\mathrm{Nu}_{\Omega} / \mathrm{Nu}_{0}=f\left(\operatorname{Re}, \mathrm{Ro}, \mathrm{Ra}^{*}, \mathrm{AR}\right)
$$

where the Reynolds number Re indicates the forced convection effect; the rotation number $\mathrm{Ro}=\operatorname{Re} \mathrm{Re}_{\Omega} / \operatorname{Re}^{2}$, a ratio of the relative strength of Coriolis force to the inertial force, represents the effects of Coriolis force on forced convection; the rotational buoyancy parameter $\mathrm{Ra}^{*}=\mathrm{Ra} \Omega / \mathrm{Re}^{2}$ denotes the effect of centrifugalbuoyancy; and the cross-sectional aspect ratio $\mathrm{AR}$ reveals the effect of the cross-sectional configuration of the rectangular duct. All the physical properties needed in calculating these parameters is evaluated at the bulk temperature $T_{b}$. The enhancement of heat transfer is presented by the ratio of the Nusselt number on rotating condition to the 


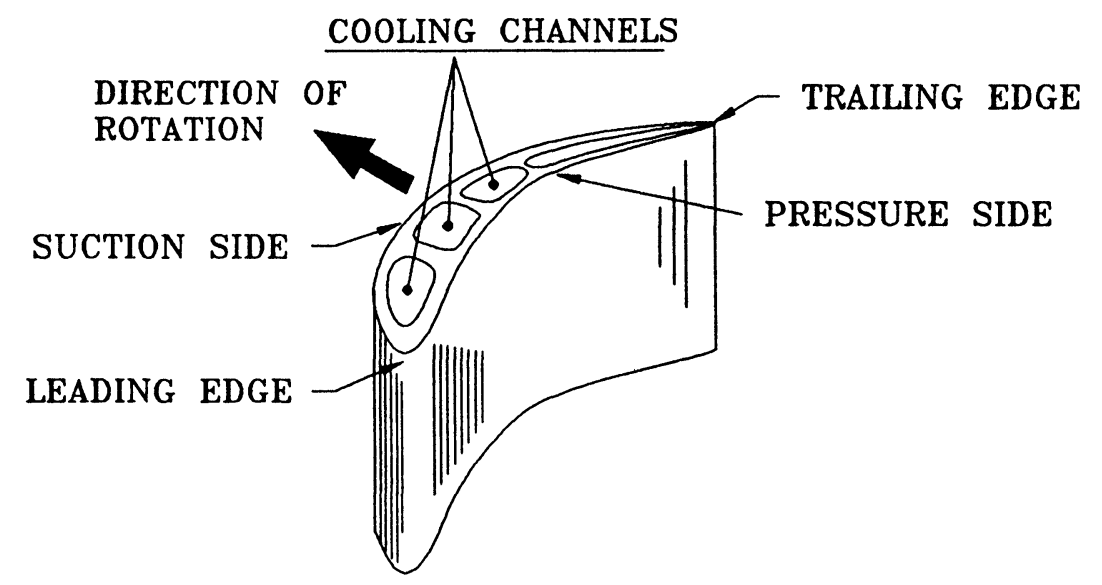

TURBINE BLADE

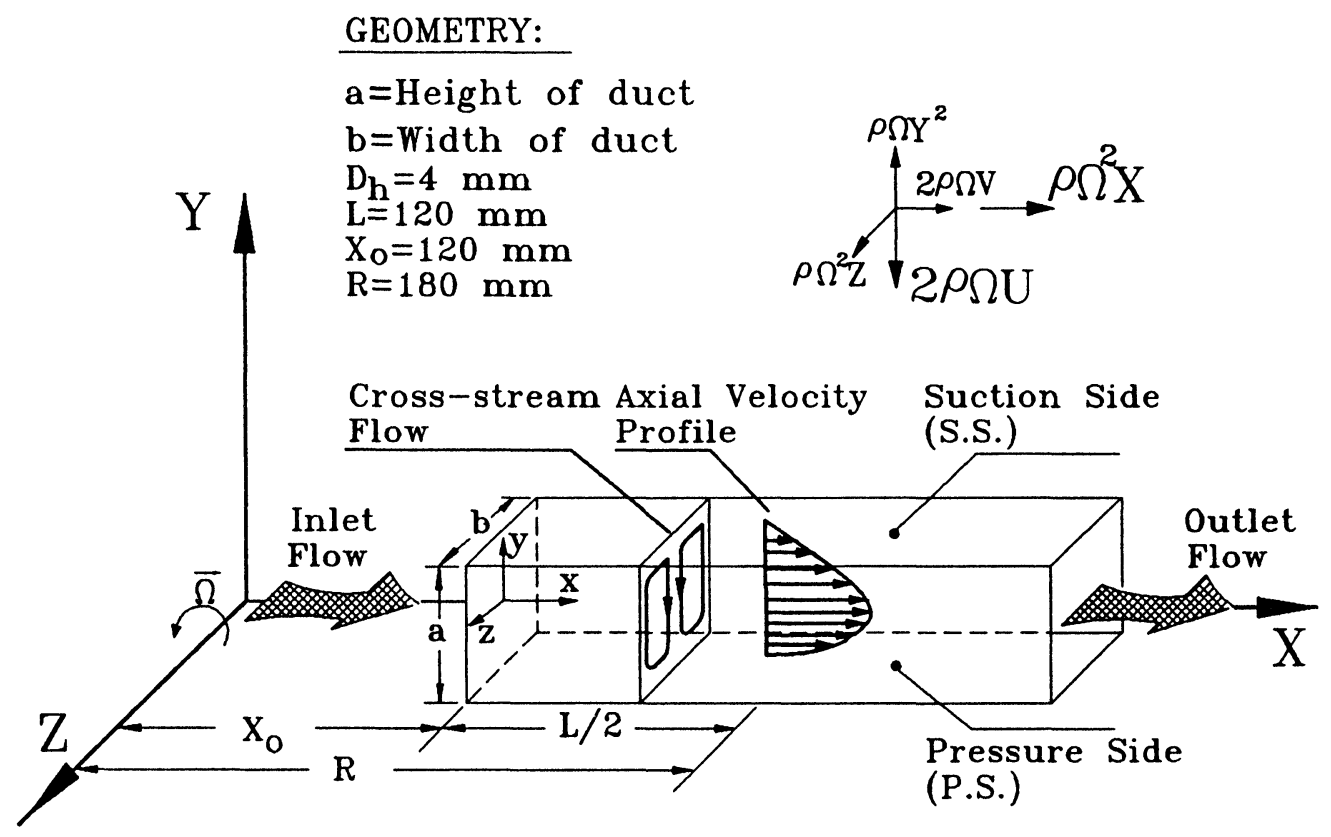

FIGURE 1 Physical model and coordinate.

corresponding nonrotating Nusselt number. The greater the Nusselt number ratio, the larger the heat transfer enhancement. On the contrary, if the Nusselt number ratio is less than unity, the heat transfer is depressed. To scale the effects of rotation and to deduce the heat transfer from the experimental data, nondimensional parameter groups were applied. Table II depicts the ranges of the experimental variables and the corresponding nondimensional parameters used in the present study.

\section{EXPERIMENTAL FACILITIES AND TEST PROCEDURE}

The experimental facilities, as illustrated in Fig. 2, consists of four major parts: coolant air supply, test section, motor with speed controller, and data acquisition system. Coolant air was supplied from a compressor through flow meters and rotary seal assembly to the test section. The flow meters of different flow ranges, from 0.2 to $8.0 \mathrm{~m}^{3} / \mathrm{hr}$, 

1. Air inlet
2. Flow meter
7. Support frame
3. Rotary joint
8. Motor
4. Slip ring
9. Tachometer
5. Safety glass
6. Test section
10. Slip ring
11. DC-power supply

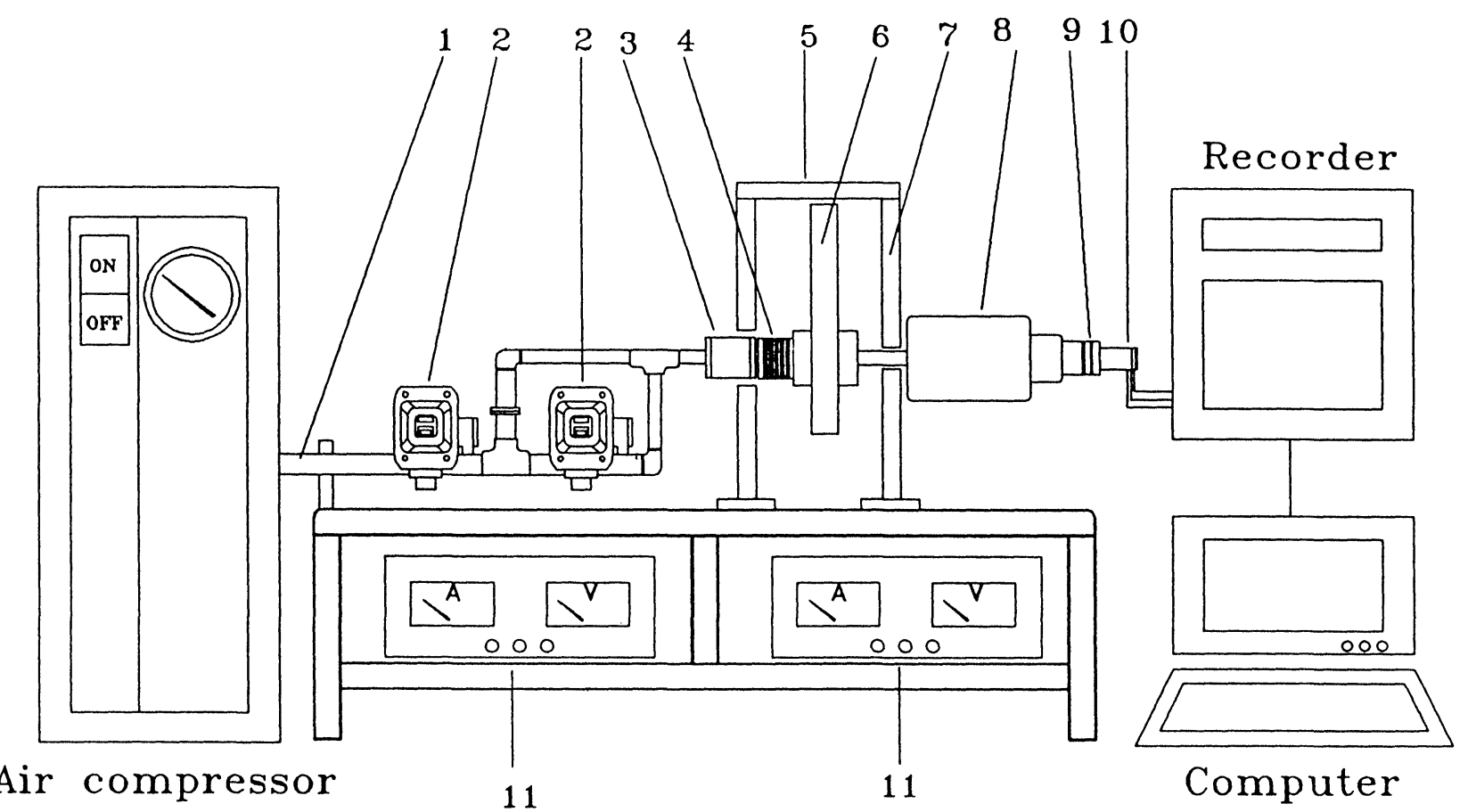

FIGURE 2 Experimental setup.

TABLE II

The experimental variables

\begin{tabular}{lc} 
& Ranges \\
\hline Variables & Radially Outward Flow \\
\hline Flow Direction & $0,500,1,000,1,500,2,000,3,000$ \\
rpm & $15,30,45$ \\
$T_{b, o}-T_{b, i}\left({ }^{\circ} \mathrm{C}\right)$ & $40-130$ \\
$T_{w}\left({ }^{\circ} \mathrm{C}\right)$ & $2.5,10.0,17.5,25.0$ \\
$x / D_{h}$ & 0.72 \\
$\operatorname{Pr}$ & $1,000,2,000,4,000,8,200,10,000,15,500$ \\
$\operatorname{Re}$ & $53.4,106.8,162.2,214.0,320.4$ \\
$\operatorname{Re}_{\Omega}$ & $0-0.32$ \\
$\operatorname{Ro}^{*}$ & $0-1.2$ \\
$\operatorname{Ra}^{*}$ & $0.5,1.0,2.0$ \\
$\mathrm{AR}^{\mathrm{AR}}$ & \\
\hline
\end{tabular}

were used for indicating the coolant flow rate. A $0.3-\mathrm{mm}$ type- $T$ thermocouple was located at the duct inlet to measure the inlet coolant bulk temperature. A mixing chamber with staggered rod bundles was attached to the exit plane for providing a well-mixed condition for outlet bulk temperature measurement by using another thermocouple set behind the mixing chamber.

Glass-fiber, reinforced plastic with a low thermal conductivity $\left(0.048 \mathrm{~W} / \mathrm{m}^{\circ} \mathrm{C}\right)$ for reducing heat loss was used for smooth duct walls. Four pieces of $0.01-\mathrm{mm}$ thickness stainless-steel film heaters, heated by electrical power supplier through slip rings, were attached separately to each interior wall surface of the duct. At a certain axial location, the wall surface temperatures were measured via thermocouples which were firmly attached to copper blocks. Morcos and Bergles [1975], Hwang and Chou [1987], and Chen and Hwang [1989] proposed wall heat conduction parameters for analyzing duct wall thermal boundary conditions: i.e.,

$$
K_{p}=\left(k_{w} t\right) /\left(k_{\mathrm{air}} D_{h}\right)
$$


TABLE III

Estimation for duct wall thermal boundary condition

\begin{tabular}{|c|c|c|c|c|c|}
\hline Material & $\begin{array}{c}k_{w} \\
\left(\mathrm{~W} / \mathrm{m}^{\circ} \mathrm{C}\right)\end{array}$ & $\stackrel{t}{t}$ & $\begin{array}{c}k_{p} \\
\text { (Based on air pure } \\
\text { conduction)* }\end{array}$ & $\begin{array}{c}k_{p} \\
\text { (Based on air mixed } \\
\text { convection) }\end{array}$ & Purpose \\
\hline Fiber Glass & 0.048 & 3.0 & 1.199 & 0.199 & wall insulation \\
\hline $\begin{array}{l}\text { Stainless } \\
\text { Film Heater }\end{array}$ & 16.3 & 0.01 & 1.357 & 0.1357 & wall insulation \\
\hline Copper block & 111.0 & 0.5 & 462.0 & 46.2 & $\begin{array}{l}\text { regional average } \\
\text { wall temperature }\end{array}$ \\
\hline
\end{tabular}

${ }^{*}$ Air thermal conductivity $k_{\text {air }}=0.03003 \mathrm{~W} / \mathrm{m}^{\circ} \mathrm{C}$

**Assumed the Nusselt number for mixed convection is 10 .

is the ratio of wall heat conduction and air pure conduction inside a duct, and

$$
K_{p}^{\prime}=\left(k_{w} t\right) /\left(h D_{h}^{2}\right)
$$

is the ratio of wall heat conduction and air mixed convection inside a duct.

Table III gives the estimated $K_{p}$ and $K_{p}^{\prime}$ values for the present test facility. Because of $K_{p}^{\prime} \ll 1.0$ for fiberglass and film heater, both of them can be considered as insulators. When the heater is supplied with electrical power, a boundary condition of nearly uniform heat flux can be achieved. On the other hand, the high $K_{p}$ and $K_{p}^{\prime}$ of the cooper block make the measurement of temperature in the block to be the regional average value.

The duct hydraulic diameter, heater active length, and the mean rotation radius were 4,120 , and $180 \mathrm{~mm}$, respectively. This gave a ratio of heater active length to hydraulic diamter of 30 , which covered most of laminar flow entrance region and both the turbulence flow entrance and fully developed regions, and a mean rotation radius to hydraulic diameter ratio of 45 , which was reasonably large as compared with the ratio in a gas turbine or an electrical machine. Further construction details of the test section are shown in Fig. 3.

The test assembly was encased in an elliptical aluminum tube with the internal void space filled with insulating ceramic cotton and was subsequently bolted on the rotating shafts so that the duct axis was perpendicular to the shaft axis. The whole model was installed in a test cell enclosed by a support frame with safety-glass plates and ventilation openings, and it was driven by a controlled electric motor. An inverter which adjusted the electric current frequency was used for controlling the rotational speed detected by a photoelectric tachometer. A slip ring located at the other end of the shaft was to transmit the detected data from the thermocouples to a recorder, as shown in Fig. 2.

\section{DATA REDUCTION}

In an experiment with either a large flow rate or a large rotational speed, the compressibility correction for the measured temperature of coolant flow must be performed. By denoting $T_{r}$ as the recovery temperature, the fluid temperature may be corrected by

$$
T=T_{r} /\left(1+\frac{k-1}{2} \gamma M^{2}\right)-\frac{(X \Omega)^{2}}{2 c_{p}}
$$

where $k$ is the specific heat ratio and $\gamma$ is the temperature recovery factor (Schlichting [1979]):

$$
\begin{array}{ll}
\gamma=\operatorname{Pr}^{1 / 2} & \text { for laminar flow } \\
\gamma=\operatorname{Pr}^{1 / 3} & \text { for turbulent flow }
\end{array}
$$

Note that in the present study a temperature correction up to $2.5^{\circ} \mathrm{C}$ was found for the case of $\operatorname{Re}=15,000(M \approx$ $0.15)$ and $1.5^{\circ} \mathrm{C}$ for $\Omega=315 \mathrm{rad} / \mathrm{s}(3,000 \mathrm{rpm})$.

At a certain axial location the heat transfer coefficient $h_{x}$ was evaluated as the ratio of the net heat flux $q_{\mathrm{net}, x}$ to the temperature difference between the heated wall temperature $T_{w, x}$ and the coolant bulk temperature $T_{b, x}$ : i.e., $h_{x}=q_{\text {net }, x} /\left(T_{w, x}-T_{b, x}\right)$. The net heat flux imposed on the coolant by convection was obtained by subtracting the external heat loss from the electric power supplied to the heater. The external heat losses were attributed to both conduction to the structure support and convection to the ambient air, and were estimated under no-flow condition by measuring heater power setting over ranges of wall temperature. The no-flow condition was achieved by filling insulation material in the channel. Based on a thermodynamic energy balance, the rise of local coolant bulk temperature was determined step by step from the inlet coolant temperature by adding the net heat flux to the coolant along the duct.

Invoking the root-sum-square method introduced by Kline and McClintock [1953] for uncertainty analysis, it 




FIGURE 3 Details of the test section.

showed in the present study that the estimated uncertainties in calculating Nusselt number were mainly affected by the local wall-to-coolant temperature and the net heat flux added to coolant from each wall. The measured variables and their uncertainties in the measurement could be expressed as: $X_{i}=X_{i}$ (measured) $\pm \delta X_{i}$, where the best estimate of $X_{i}$ is $X_{i}$ (measured) and there was an uncertainty in $X_{i}$ that might be as large as $\delta X_{i}$. For the case of $\mathrm{AR}=1.0$ and $\operatorname{Re}_{\Omega}=162.2$, Fig. 4 showed the typical variations of local wall-to-coolant bulk temperature along the test duct for $\operatorname{Re}=1000, q_{\text {net }}=1,150 \mathrm{~W} / \mathrm{m}^{2}$, and $\mathrm{Re}=$ $10,000, q_{\text {net }}=10,500 \mathrm{~W} / \mathrm{m}^{2}$, respectively. Uncertainty in the Nusselt number increased with the decrease in either the wall-to-coolant temperature difference or the net heat flux. It was found that the largest uncertainty of 20 percent was observed for $\operatorname{Re}=1,000$ at $x / D_{h}=25.0$ on the pressure side because of the corresponding low wall-tocoolant bulk temperature difference and low heat flux. The uncertainty in the Nusselt numbers was approximately 8 percent when Reynolds number was greater than 10,000 . 


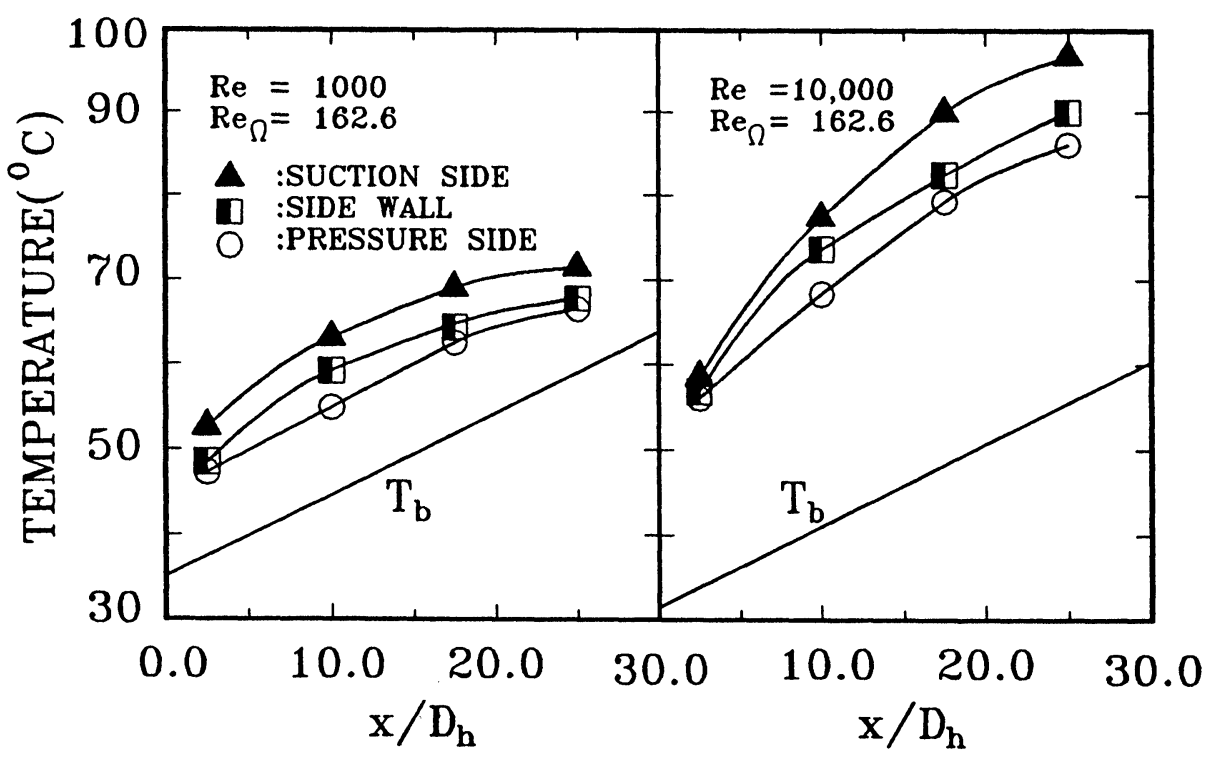

FIGURE 4 Wall and coolant temperature variations along the test section in the case of $A R=1.0$.

\section{RESULTS AND DISCUSSION}

The thermocouples were installed on two adjacent walls of the channel only. By rotating the radial channel, clockwise or counter-clockwise or switching the channel 90 degrees, one was able to obtain the data on pressure side, suction side, and two side walls or for $\mathrm{AR}=0.5$, and 2.0. Experiments were first conducted to determine the regional average Nusslet numbers for the nonrotating case along the four duct sides for a range of Reynolds numbers $(\operatorname{Re}=1,000 \sim 15,000)$, positions $\left(x / D_{h}=2.5,10.0\right.$, 17.5, and 25.0), and outlet-to-inlet temperature differences $\left(T_{b, o}-T_{b, i}=15.0,30.0\right.$, and $\left.45.0^{\circ} \mathrm{C}\right)$. For the case of $\mathrm{AR}=1.0$, Fig. 5 gives the results of nonrotating condition which are compared with the Dittus-Boelter [1930] correlation in the turbulent flow regime and the Perkins et al. [1973] correlation in the laminar flow regime, respectively. The correlations are

\section{Dittus-Boelter [1930]}

$$
\mathrm{Nu}_{0}=\mathrm{Nu}_{\mathrm{TFD}}\left[1.0+2.0 /\left(x / D_{h}\right)\right]
$$

for $x / D_{h} \geq 10.0$, where $\mathrm{Nu}$ TFD $=0.023 \mathrm{Re}^{0.8} \mathrm{Pr}^{0.4}$ is for fully developed flow in a circular duct with a uniform wall temperature, and
Perkins et al. [1973]

$$
\mathrm{Nu}_{0}=1 /[0.277-0.152 \exp (-38.6 \delta)]
$$

for square duct with a uniform wall heat flux, where $\delta=x /\left(D_{h} \operatorname{Re} \operatorname{Pr}\right) \geq 0.005$. Fig. 5 shows that for the higher Reynolds number the Nusselt number is approximately within 10 percent of that of Dittus-Boelter [1930]. However, the higher Nusselt number at $x / D_{h}=25.0$ was affected by the discontinuity of the uniform heat flux thermal boundary condition at the duct exit region.

Figures 6(a), (b) and (c) indicate the effects of rotation on heat transfer along the test section for selected through-flow Reynolds numbers with aspect ratios 0.5 , 1.0 , and 2.0, respectively. It is noted that the Nusselt number ratios at the pressure side were always greater than those at the suction side but this trend was attenuated with the higher Reynolds number. This was in agreement with the results of Mori et al. [1968, 1971] that rotational heat transfer enhancement for laminar flow, in general, was more prominent than that for turbulent flow. The different behaviors on heat transfer over the pressure side and the suction side were due to the Coriolis-induced secondary cross streams in the form of a vortex pair which impinged toward the pressure side, then caused a return flow which carried already heated, relatively quiescent fluid from the pressure side and side walls to near the suction side. At higher rotation rate the strength of the Coriolis-induced 

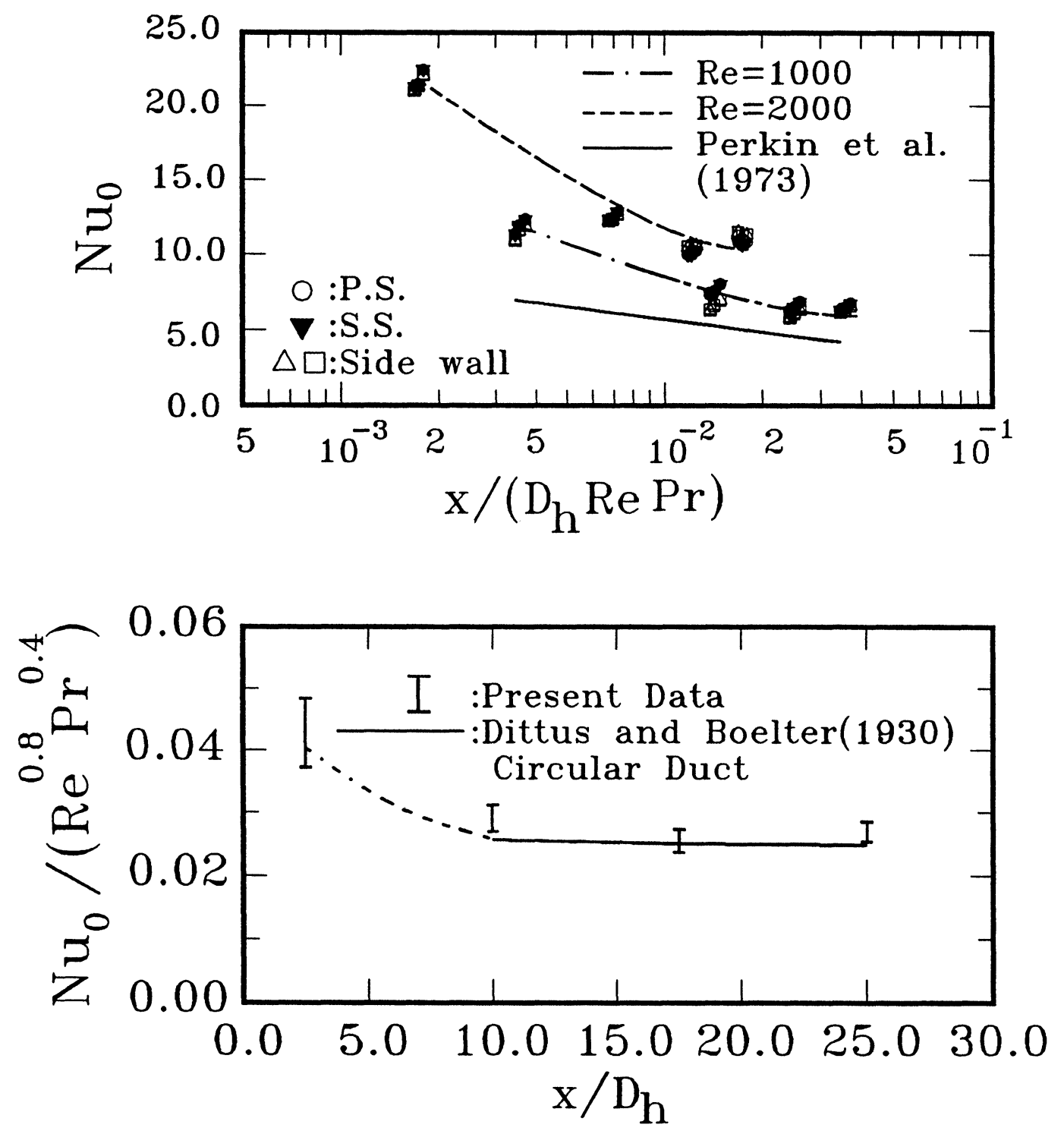

FIGURE 5 Comparison of $\mathrm{Nu}$ in laminar and turbulent flow regime for $\mathrm{AR}=1.0$.

cross streams was more intensified and this trend was more noticeable than that at lower rotation rate. It is also seen that the Nusselt number ratios at both pressure and suction sides dropped near duct outlet for most cases under study. This was due to the increase of $\mathrm{Nu}_{0}$ near exit as shown in Fig. 5. For the effect of duct aspect ratio, large aspect ratio (long side aligned with the Coriolis force) gave larger short side direction Coriolis force gradient, but yielded a greater cross-sectional flow resistance. Due to the combination of these two effects, the largest heat transfer enhancement was seen for the case of $A R=1.0$, and the enhancement for $\mathrm{AR}=0.5$ is greater than that for $\mathrm{AR}=2.0$. For some cases, the heat transfer was depressed on the suction side because of the stabilizing effect of the vortex motion on the main flow disturbances.

Considering the effect of the Coriolis-induced cross streams on the main flows, Fig. 7 discloses the variations of the Nusselt number ratios with rotation number for the case of aspect ratio of 1.0, along with a comparison to the experimental results by Han and Zhang [1992]. The results show that both the pressure side and suction side Nusselt number ratios of the present study at $x / D_{h}=10.0$ agreed fairly with those of Han and Zhang [1992] at $x / D_{h}=9.0$ and 11.0. Note that the results of Han and Zhang [1992] were based on the following conditions: $R o$ calculated at rotational speed $=400$ and $800 \mathrm{rpm}$, Re between 2,500 


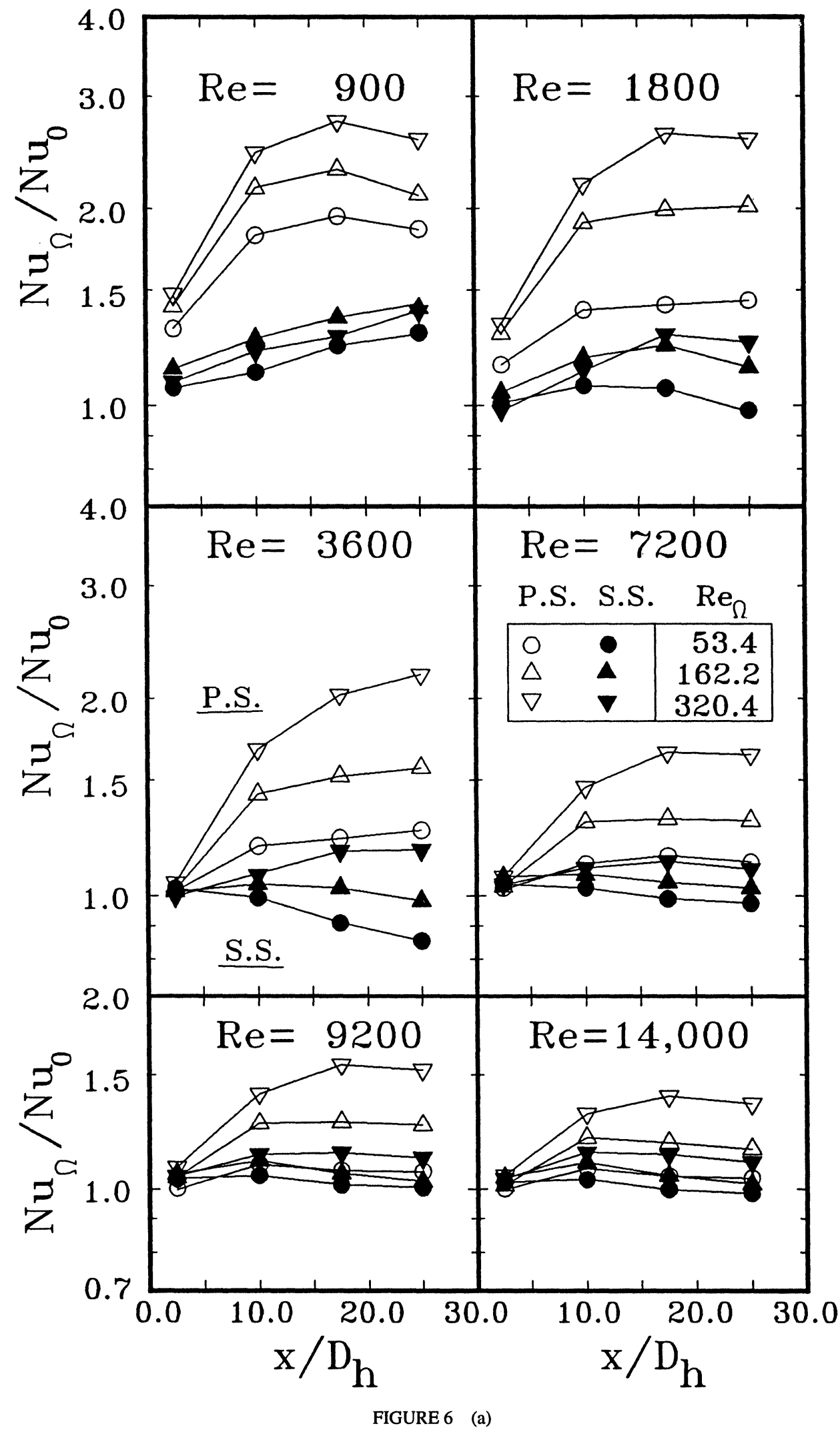




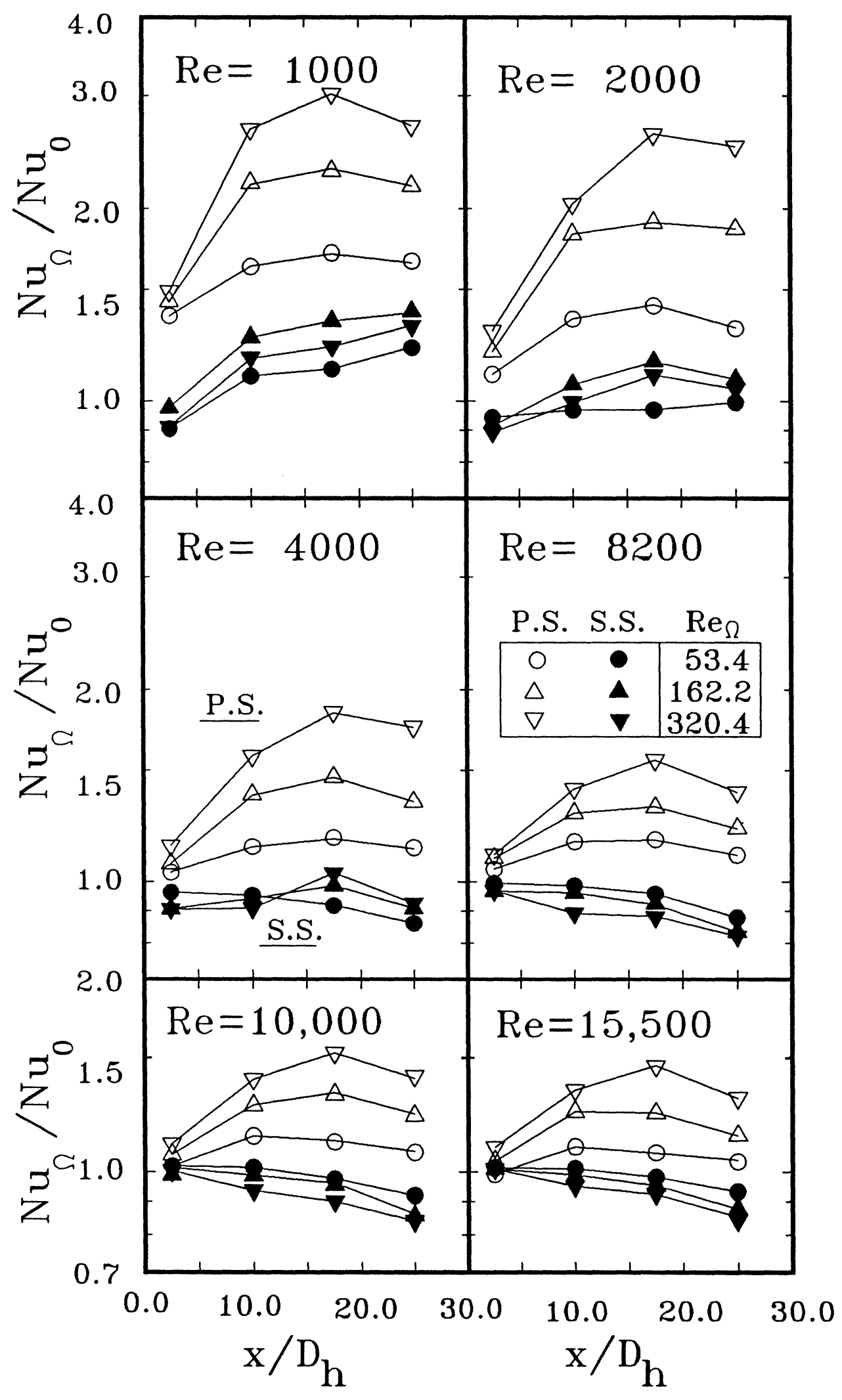

FIGURE 6 (b) 


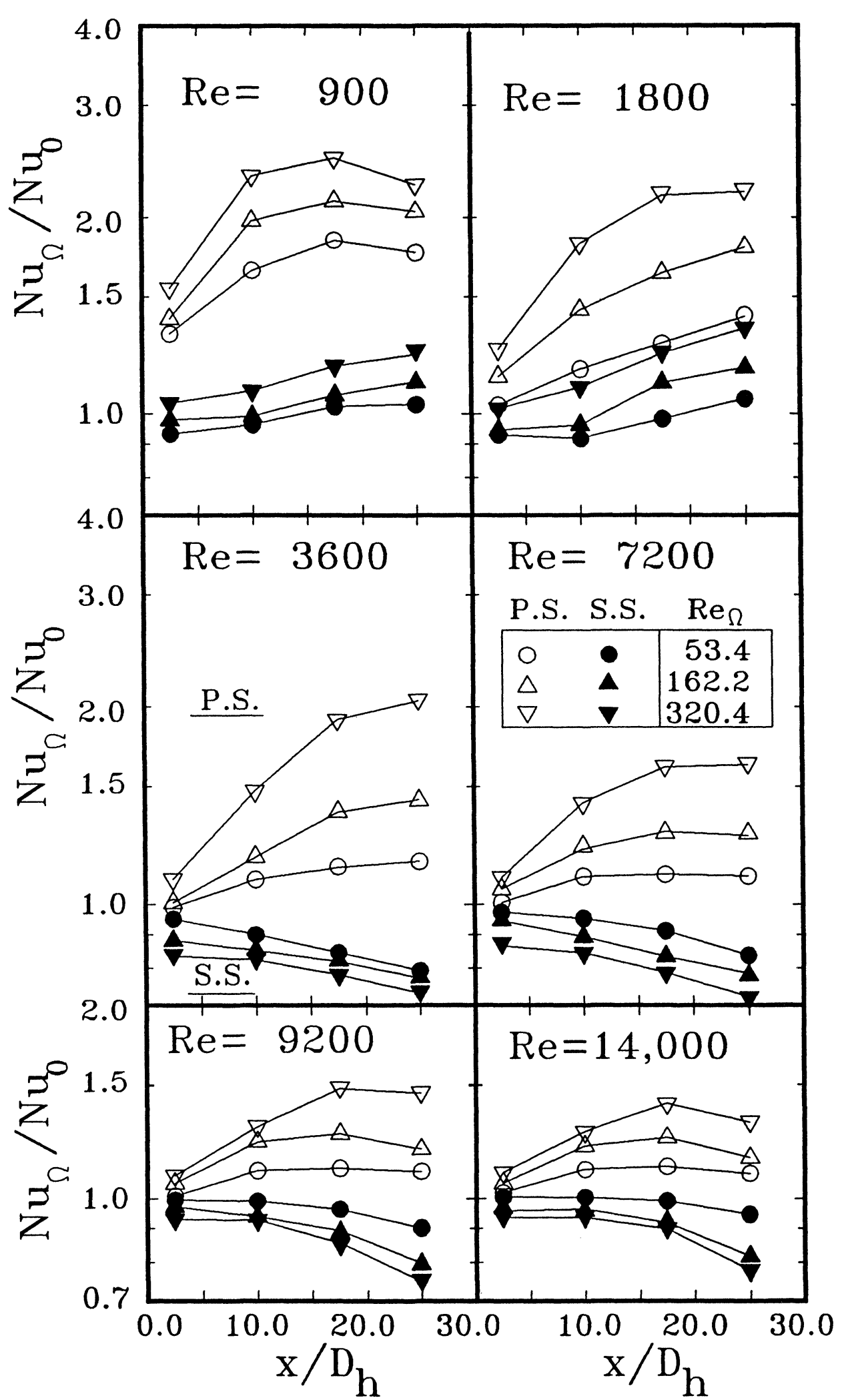

FIGURE 6 (c) Variations of $\mathrm{Nu}_{\Omega} / \mathrm{Nu}_{0}$ with $x / D_{h}$ based on $T_{b, o}-T_{b, i}=30.0^{\circ} \mathrm{C}$ for the cases of aspect ratios of (a) 0.5 , (b) 1.0, and (c) 2.0 . 


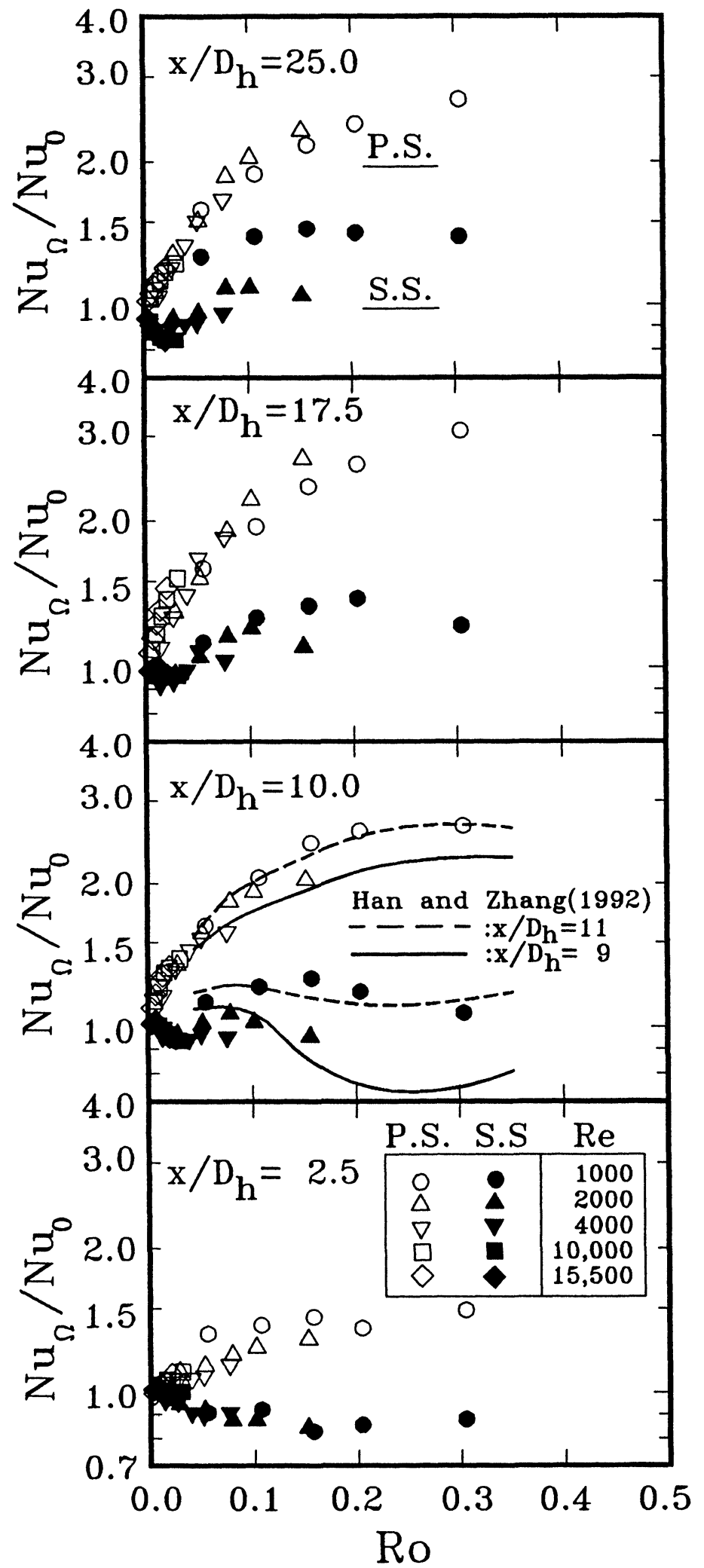

FIGURE 7 Variations of $\mathrm{Nu}_{\Omega} / \mathrm{Nu}_{0}$ from $R o$ for $\mathrm{AR}=1.0$ and $T_{b, o}-T_{b, i}=30.0^{\circ} \mathrm{C}$. 
and 25,000, $R / D_{h}=30$, and $D_{h}=12.7 \mathrm{~mm}$. The present data were based on: $R o$ calculated at rotational speed $=$ $500,1,500$, and 3,000 rpm, Re between 1,000 and 15,500, $R / D_{h}=45$, and $D_{h}=4 \mathrm{~mm}$. This confirms that $R o$ is indeed an important heat transfer governing parameter in a rotating channel. By either increasing the rotational speed or decreasing the Reynolds number, a higher Ro can be achieved. In the entry region, $x / D_{h}=2.5$, it is also seen that the observed enhancement in heat transfer for the present developing flow was less than that of higher $x / D_{h}$. This result was consistent with the experiment of Metzger and Stan [1977] for entry region heat transfer in a rotating radial tube.

To investigate the geometry effect of cross-sectional aspect ratio, Fig. 8 demonstrates the influence of aspect ratio $0.5,1.0$, and 2.0 on the Nusselt number ratios. The results show that the enhancement of the Nusselt number ratios for the case of $\mathrm{AR}=1.0$ was always highest, and the enhancement for the case of $A R=0.5$ was higher than that for $\mathrm{AR}=2.0$. This was due to the combination effect of weak long side Coriolis force gradient for low AR and high flow resistance for high AR. These phenomena can also be observed in heated horizontal rectangular ducts (Cheng and Hwang [1969] and curved channels (Cheng and Akiyama [1970]. The depression of heat transfer on the suction side was also seen for small $R o$ case.

By definition the rotational buoyancy parameter is affected by the rotation number, wall-to-coolant temperature, eccentricity, and local positions. To highlight the salient feautres of the centrifugal-buoyant radial secondary flows, three outlet-to-inlet bulk temperature differences, $T_{b, o}-T_{b, i}=15.0,30.0$, and $45.0^{\circ} \mathrm{C}$, were selected while other operating parameters were held constant during each measurement. Figs. 9(a), (b), and (c) illustrate the results of the variations of Nusselt number ratios with rotational buoyancy parameters at axial location of $x / D_{h}=17.5$ and for aspect ratios $0.5,1.0$, and 2.0, respectively. As the rotation number was fixed, it was found that increasing the rotational buoyancy parameter decreased the Nusselt number ratios at both the pressure side and suction side for low Reynolds number flows $\mathrm{Re} \cong 1,000$, but the trend was reversed for $\operatorname{Re} \cong 4,000$. Then, these trends were diminished for higher Reynolds number. These phenomena can be found from analyzing the mixed convection of the buoyancy-induced opposing flows in a vertical heated tube for both constant wall temperature and uniform wall heat flux: the buoyancy forces tend to decrease the laminar heat transfer rate while they increase the turbulent heat transfer rate (Abdelmeguid and Spalding [1979]; Buhr et al. [1974]. With increasing the rotational buoyance parameters, the depressed effect on heat transfer agree with those proposed by Morris and $\mathrm{Ay}$ - han [1979], Clifford et al. [1984], Harasgama and Morris [1988], and Soong et al. [1991]; on the other hand, the increased tendencies on heat transfer were found by Wagner et al. [1991a, b], Morris and Ghvami-Nasr [1991], and Han and Zhang [1992]. It is seen that the depression of Nusselt number for the centrifugal buoyancy force is bigger for the cases of $A R=0.5$ and 2.0 than that for AR $=1.0$. One may attribute this phenomenon to the larger peripheral area of $A R=0.5$ and 2.0 for more heated fluid with decelerated axial velocity.

\section{PRACTICAL IMPORTANCE}

1. The study of internal convective cooling in rotating ducts is of engineering importance for its applications to the cooling of turbine blades and cooling of electrical machinery.

2. Rectangular ducts of aspect ratios other than 1.0 may be applied to the internal cooling passage near the trailing edge of a turbine blade and in the cooling passage in a rotor of electrical machinery.

\section{CONCLUSION}

The investigation has presented rotation effects, Coriolisinduced cross streams, and centrifugal-buoyant radial secondary flows, on convective heat transfer of radially outward flows in rotating rectangular ducts with $\mathrm{AR}=0.5$, 1.0, and 2.0. According to an analysis with a wall heat conduction parameter $\left(K_{p}\right)$, four pieces of stainless-steel film heater of $0.01-\mathrm{mm}$ thickness were separately lined with the interior wall surfaces of the fiberglass duct to obtain the nearly uniform wall heat flux boundary conditions. The results obtained and described in this experiment are presented as follows.

1. Due to rotation, the Coriolis-induced cross streams impinge directly toward the pressure side, then cause a return flow which carriers already heated, relatively quiescent fluid from the pressure side and side walls to near the suction side. Therefore, the Coriolis-induced cross streams create additional mixing to the main flows and enhance the heat transfer rate, especially at the pressure side. Also, the enhancement of heat transfer rate is gradually attenuated with increasing through-flow Reynolds number because of the effect of the turbulence becoming progressively larger than that induced by rotation.

2. The rotation number, effect of the Coriolis-induced cross streams on the forced main flows, performs an im- 


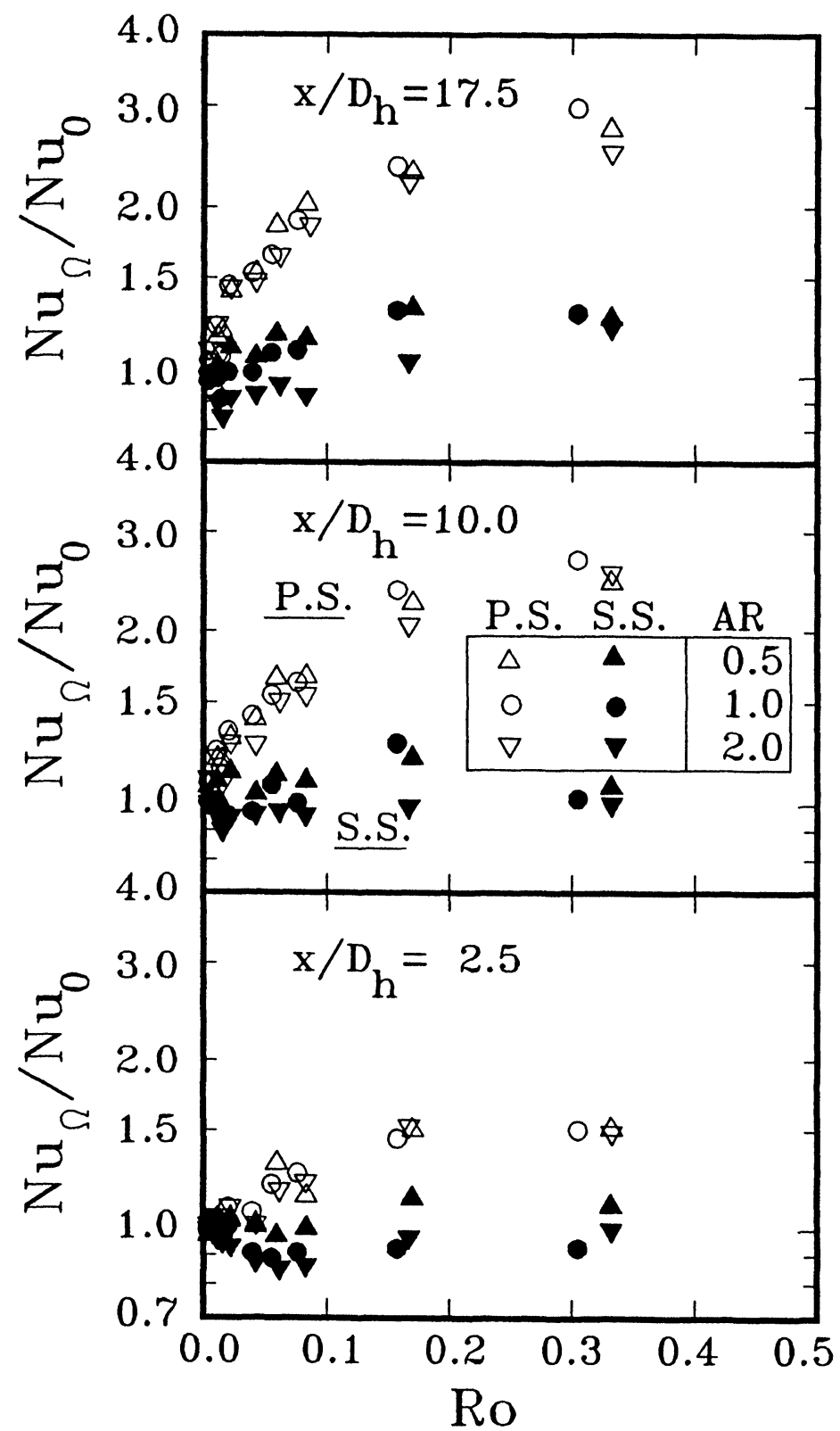

FIGURE 8 Variations of $\mathrm{Nu}_{\Omega} / \mathrm{Nu}_{0}$ from $R o$ for the cases of different aspect ratio

portant parameter to the internal convective heat transfer for radially rotating duct flows and a higher value can be obtained by either increasing the rotational speed or decreasing the through-flow Reynolds number. The higher the rotation number, the more intensified the strength of the Coriolis-induced cross streams and the more noticeable the enhancement of the heat transfer rate.
3. For high aspect ratio narrow duct, the short side Coriolis force gradient is large, but the Coriolis-induced cross streams are weakened by viscous force over the longer side walls. Therefore, due to the combination of these two effects, the heat-transfer enhancement on the pressure side for the largest for $\mathrm{AR}=1.0$ and the enhancement for $\mathrm{AR}$ $=0.5$ are larger than that for $A R=2.0$. For some cases, the heat transfer is depressed on the suction side because 


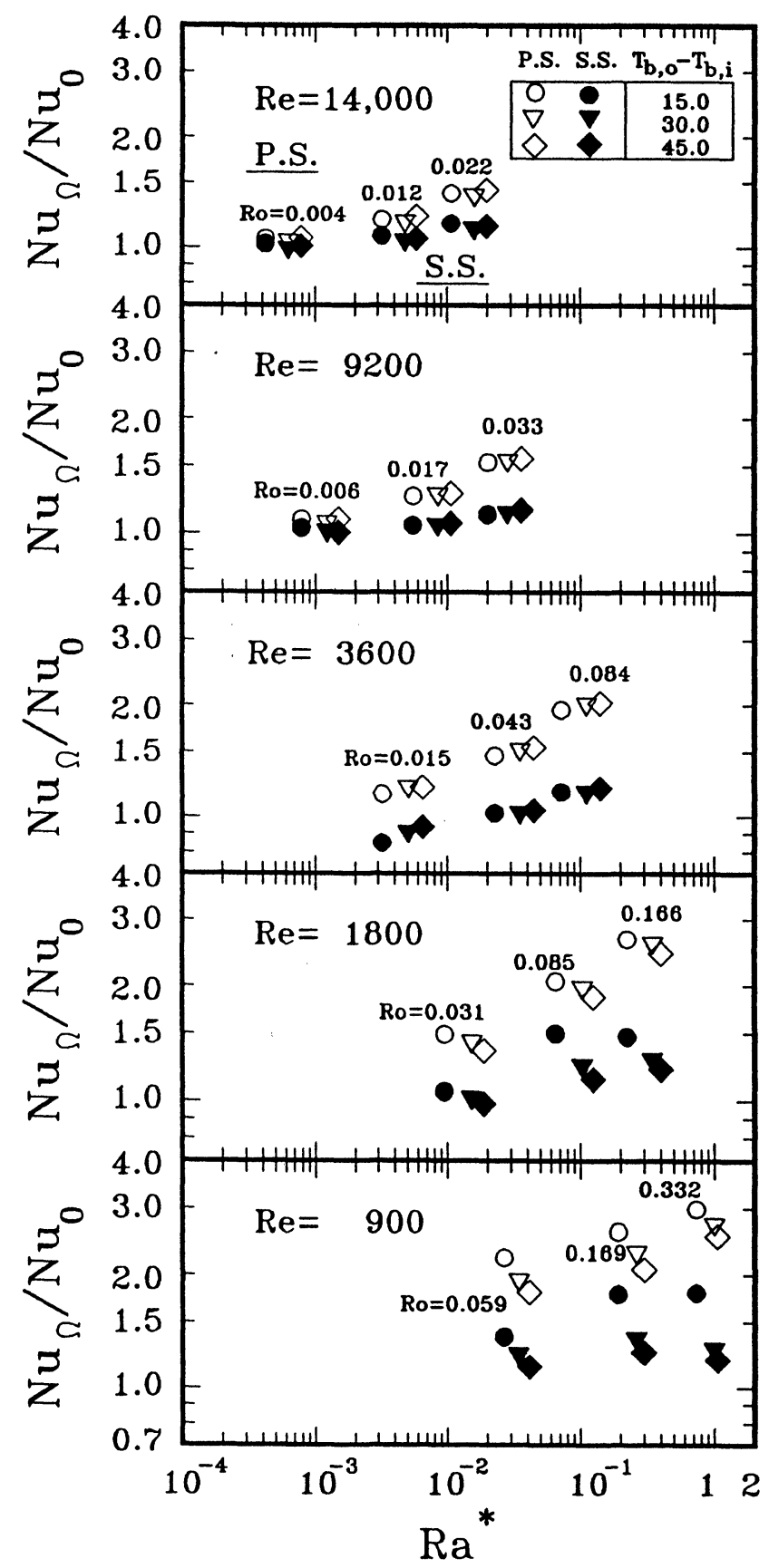

FIGURE 9 (a)

of the stabilizing effect of the vortex motion on the main flow disturbances.

4. Varying the difference of the outlet-to-inlet air bulk temperature while other operating parameters were held constant during the experiment, the increasing rotational buoyancy parameters made the heat transfer rate decrease for cases of $\operatorname{Re} \cong 1,000$ but increase for cases of $\operatorname{Re} \cong 4,000$, and these trends are then diminished for higher Reynolds numbers. These phenomena can be found for buoyancy-induced opposing flows in a vertical heated tube: the buoyancy effect decreases the laminar heat transfer rate but increases the turbulent heat transfer rate, and this trend is more pronounced for $\mathrm{AR}=0.5$ and 2.0 than that for $\mathrm{AR}=1.0$. 


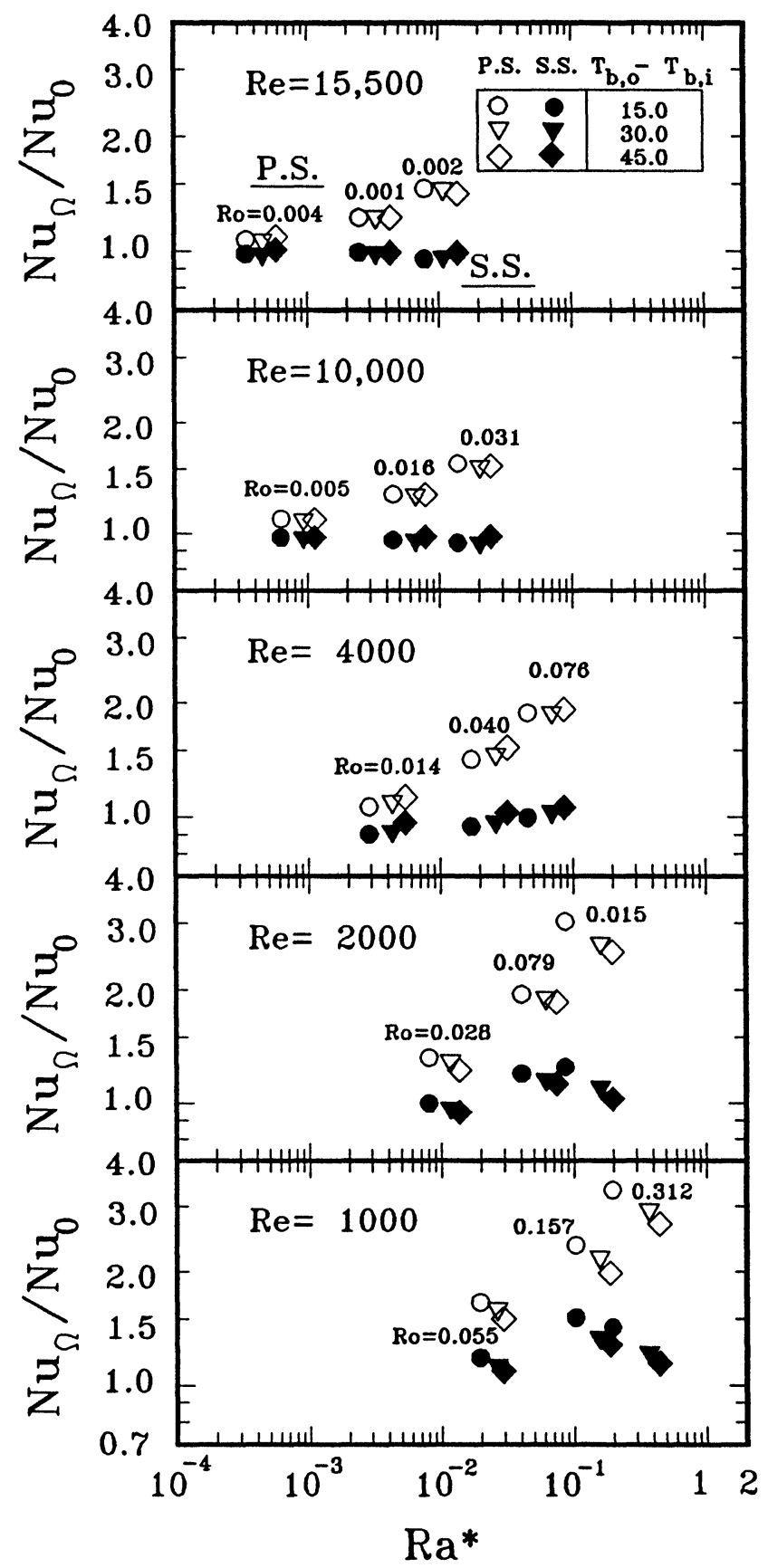

FIGURE 9 (b)

\section{Nomenclature}

$a \quad$ height of rectangular duct

AR cross-sectional aspect ratio $=a / b$

$b \quad$ width of rectangular duct

$c_{p} \quad$ specific heat of air, $\mathrm{J} /\left(\mathrm{kg}^{\circ} \mathrm{C}\right)$

$D_{h} \quad$ hydraulic diameter $=2 a b /(a+b)$

$h_{x} \quad$ local heat transfer coefficient $=q_{\text {net, } x} /\left(T_{w, x}-T_{b, x}\right)$, $\mathrm{W} /\left(\mathrm{m}^{2 \circ} \mathrm{C}\right)$ $k_{\text {air }} \quad$ thermal conductivity of air, $\mathrm{W} /\left(\mathrm{m}^{\circ} \mathrm{C}\right)$

$K_{p} \quad$ wall heat conduction parameter $=k_{w} t /\left(k_{\mathrm{air}} D_{h}\right)$

$k_{w} \quad$ thermal conductivity of wall, $\mathrm{W} /\left(\mathrm{m}^{\circ} \mathrm{C}\right)$

$L \quad$ actively heated length of duct, $\mathrm{mm}$

$M \quad$ mach number

$\mathrm{Nu} \quad$ Nusselt number $=h_{x} D_{h} / k_{\text {air }}$

$\mathrm{Nu}_{o} \quad$ Nusselt number for nonrotating condition

$\mathrm{Nu}_{\Omega} \quad$ Nusselt number for rotating condition

P.S. pressure side 


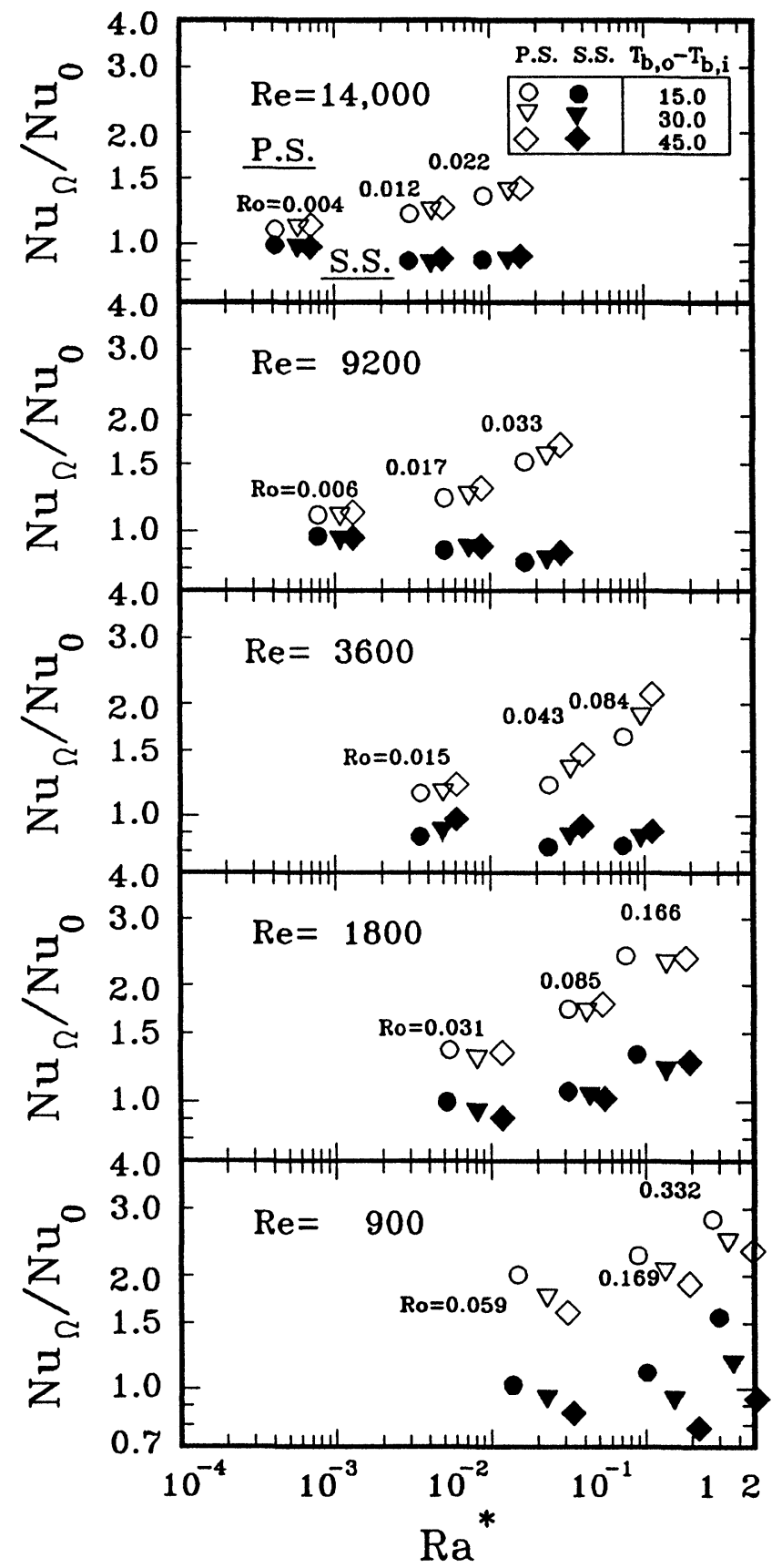

FIGURE 9 (c) Effects of $\mathrm{Ra}^{*}$ on $\mathrm{Nu}_{\Omega} / \mathrm{Nu}_{0}$ for the cases of aspect ratios of (a) 0.5 , (b) 1.0, and (c) 2.0 .

$\begin{array}{ll}\operatorname{Pr} & \text { Prandtl number }=c_{p} \mu / k_{\text {air }} \\ q_{\text {net }} & \text { net wall heat flux, W/m } \\ R & \text { mean rotation radius }=X_{o}+L / 2, \mathrm{~mm} \\ \operatorname{Ra} \Omega & \text { rotational Rayleigh number }= \\ & \operatorname{Ro}^{2} \operatorname{Re}^{2}\left(\varepsilon+x / D_{h}\right)\left[\left(T_{w, x}-T_{b, x}\right) / T_{b, x}\right] \operatorname{Pr} \\ \mathrm{Ra}^{*} & \text { rotational buoyancy parameter }=\operatorname{Ra}_{\Omega} / \mathrm{Re}^{2} \\ \operatorname{Re} & \text { through-flow Reynolds number }=\rho U_{o} D_{h} / \mu \\ \mathrm{Re}_{\Omega} & \text { rotational Reynolds number }=\rho \Omega D_{h}^{2} / \mu \\ \operatorname{Ro} & \text { Rotation number }=\operatorname{Re}_{\Omega} / \operatorname{Re}=\Omega D_{h} / U_{o}\end{array}$

$\begin{array}{ll}\text { S.S. } & \text { suction side } \\ t & \text { wall thickness, mm } \\ T_{b} & \text { bulk temperature of air, }{ }^{\circ} \mathrm{C} \\ T_{b, i} & \text { inlet air bulk temperature, }{ }^{\circ} \mathrm{C} \\ T_{b, o} & \text { outlet air bulk temperature, }{ }^{\circ} \mathrm{C} \\ T_{b, x} & \text { air bulk temperature at local position } x,{ }^{\circ} \mathrm{C} \\ T_{r} & \text { recovery temperature of air, }{ }^{\circ} \mathrm{C} \\ T_{w, x} & \text { duct wall temperature at } x,{ }^{\circ} \mathrm{C} \\ U_{o} & \text { mean air velocity, } \mathrm{m} / \mathrm{s}\end{array}$




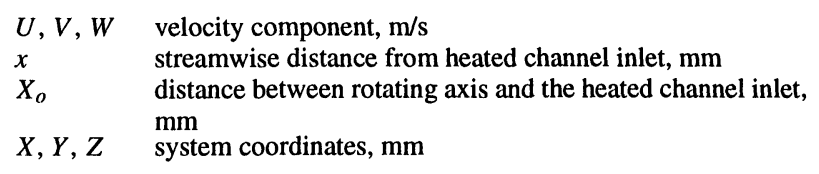

\section{Greek letters}

$\begin{array}{ll}\varepsilon & \text { eccentricity }=X_{o} / D_{h} \\ \gamma & \text { temperature recovery factor } \\ \mu & \text { air dynamic viscosity, } \mathrm{kg} / \mathrm{ms} \\ \rho & \text { air density, } \mathrm{Kg} / \mathrm{m}^{3} \\ \Omega & \text { rotational speed, } \mathrm{rad} / \mathrm{s}\end{array}$

\section{References}

Abdelmeguid, A. M., and Spalding, D. B., 1979. Turbulent Flow and Heat Transfer in Pipes with Buoyancy Effects, Journal of Fluid Mechanics, Vol. 94, pp. 383-400.

Barua, S. N., 1955. Secondary Flow in a Rotating Straight Pipe, Proceedings of the Society of Royal London, Vol. 227 A, pp. 133-139.

Buhr, H. O., Horsten, E. A., and Carr, A. D., 1974. The Distortion of Turbulent Velocity and Temperature Profiles on Heating for Mercury in a Vertical Pipe, Transactions of the ASME Journal of Heat Transfer, Vol. 96, pp. 152-158.

Chen, R. S., and Hwang, G. J., 1989. Effect of Wall Conduction Combined Free and Forced Laminar Convection in Horizontal Tubes, Transactions of the ASME Journal of Heat Transfer, Vol. 111, No. 2, pp. 581-585.

Cheng, K. C., and Akiyama, M., 1970. Laminar Forced Convection Heat Transfer in Curved Rectangular Channels, International Journal of Heat and Mass Transfer, Vol. 13, pp. 471-490.

Cheng, K. C., and Hwang, G. J., 1969. Numerical Solution for Combined Free and Forced Laminar Convection in Horizontal Rectangular Channels, Transactions of the ASME Journal of Heat Transfer, Vol. 91, pp. 59-66.

Clifford, R. J., Harasgama, S. P., and Morris, W. D., 1984. An Experimental Study of Local and Mean Heat Transfer in a Triangular-Sectioned Duct Rotating in the Orthogonal Mode, Transactions of the ASME Journal of Engineering for Gas Turbines and Power, Vol. 106, pp 661-667.

Dittus, P. W., and Boelter, L. M. K., 1930. Heat Transfer in Automobile Radiators of the Tubular Type, University of California, Publications in Engineering, Vol. 2, No. 13, pp. 443-461; reprinted in 1985, Int Comm. Heat Mass Transfer, Vol. 12, pp. 3-22.

Guidez, J., 1989. Study of the Convective Heat Transfer in a Rotating Coolant Channel, Transactions of the ASME Journal of Turbomachinery, Vol. 111, pp. 43-50.

Han, J. C., and Zhang, Y., 1992. Effect of Uneven Wall Temperature on Local Heat Transfer in a Rotating Square Channel with Smooth Walls and Radial Outward flow, Transactions of the ASME Journal of Heat Transfer, Vol. 114, pp. 850-858.
Harasgama, S. P., and Morris, W. D., 1988. The Influence of Rotation on the Heat Transfer Characteristics of Circular, Triangular, and SquareSectioned Coolant Passage of Gas Turbine Rotor Blades, Transactions of the ASME Journal of Turbomachinery, Vol. 110, pp. 44-50.

Hwang, G. J., and Chou, F. C., 1987. Effect of Wall Conduction on Combined Free and Forced Laminar Convection in Horizontal Rectangular Channels, Transactions of the ASME Journal of Heat Transfer, Vol. 109, pp. 936-942.

Hwang, G. J., and Kuo, C. R., 1993. Experimental Study of Convective Heat Transfer in a Rotating Square Duct with Radially Outward Flow, 1993 National Heat Transfer Conirerence, Atlanta, GA, August 8-11.

Hwang, G.J., and Soong, C.Y., 1989. Experimental Automation and Heat Transfer Measurement on a Rotating Thermal System, Transport Phenomena in Thermal Control, edited by G. J. Hwang, Hemisphere Publishing, New York, pp. 375-388.

Kline, S. J., and McClintock, F. A., 1953. Describing Uncertainties in Single Sample Experiments, Mechanical Engineering, Jan., pp. 3-8.

Metzger, D. E. and Stan, R. L., 1977. Entry Region Heat Transfer in Rotating Radial Tubes, AIAA Paper No. 77-189, presented at the 15th AIAA Aerospace Sciences Meeting, Los Angeles, CA.

Morcos, S. M., and Bergles, A. E., 1975. Experimental Investigation of Combined Forced and Free Laminar Convection in Horizontal Tubes, Transactions of the ASME Journal of Heat Transfer, Vol. 97, pp. 212-219.

Mori, Y., Fukada, T., and Nakayama, W., 1968. Convective Heat Transfer in a Rotating Circular Pipe (1st Report, Laminar Region). International Journal of Heat and Mass Transfer, Vol. 11, pp. 1027-1040.

Mori, Y., Fukada, T., and Nakayama, W., 1971. Convective Heat Transfer in a Rotating Circular Pipe (2nd Report), International Journal of Heat and Mass Transfer, Vol. 14, pp. 1807-1824.

Morris, W. D., and Ayhan T., 1979. Observation on the Influence of Rotation on Heat Transfer in the Coolant Channels of Gas Turbine Rotor Blades, Proceedings of the Institute of Mechanical Engg., Vol. 193, pp. 303-311.

Morris, W. D. and Ghavami-Nasr, G., 1991. Heat Transfer in Rectangular Channel with Orthogonal Mode Rotation, Transactions of the ASME Journal of Turbomachinery, Vol. 113, pp. 339-345.

Perkins, K. R., Shade, K. W., and McEligot, D. M., 1973. "Heat Laminarizing Gas Flow in a Square Duct, International Journal of Heat and Mass Transfer, Vol. 16, pp. 897-976.

Schlichting, H., 1979. Boundary Layer Theory, 7th ed., McGraw-Hill, New York, pp. 335, 714

Soong, C. Y., Lin, S. T. and Hwang, G. J., 1991. An Experimental Study of Convective Heat Transfer in Radially Rotating Rectangular Ducts, Transactions of the ASME Journal of Heat Transfer, Vol. 113, pp. 604-611.

Wagner, J. H., Johnson, B. V., and Hajek, T. J., 1991a. Heat Transfer in Rotating Passages with Smooth Walls and Radial Outward Flow, Transactions of the ASME Journal of Turbomachinery, Vol. 113, pp. 42-51.

Wagner, J. H., Johnson, B. V., and Kopper, F. C., 1991b. Heat Transfer in Rotating Serpentine Passages with Smooth Walls, Transactions of the ASME Journal of Turbomachinery, Vol. 113, pp. 321-330. 

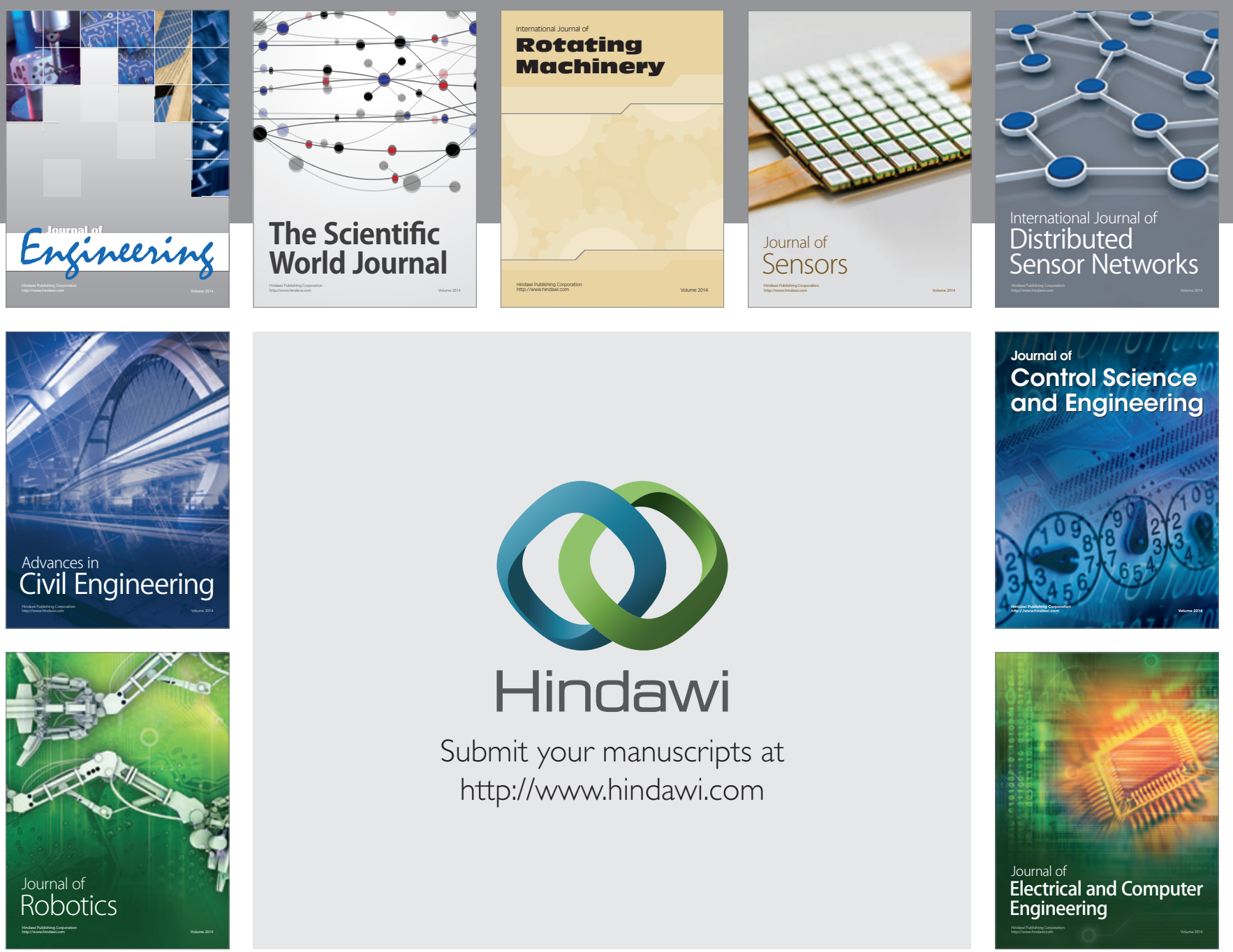

Submit your manuscripts at

http://www.hindawi.com
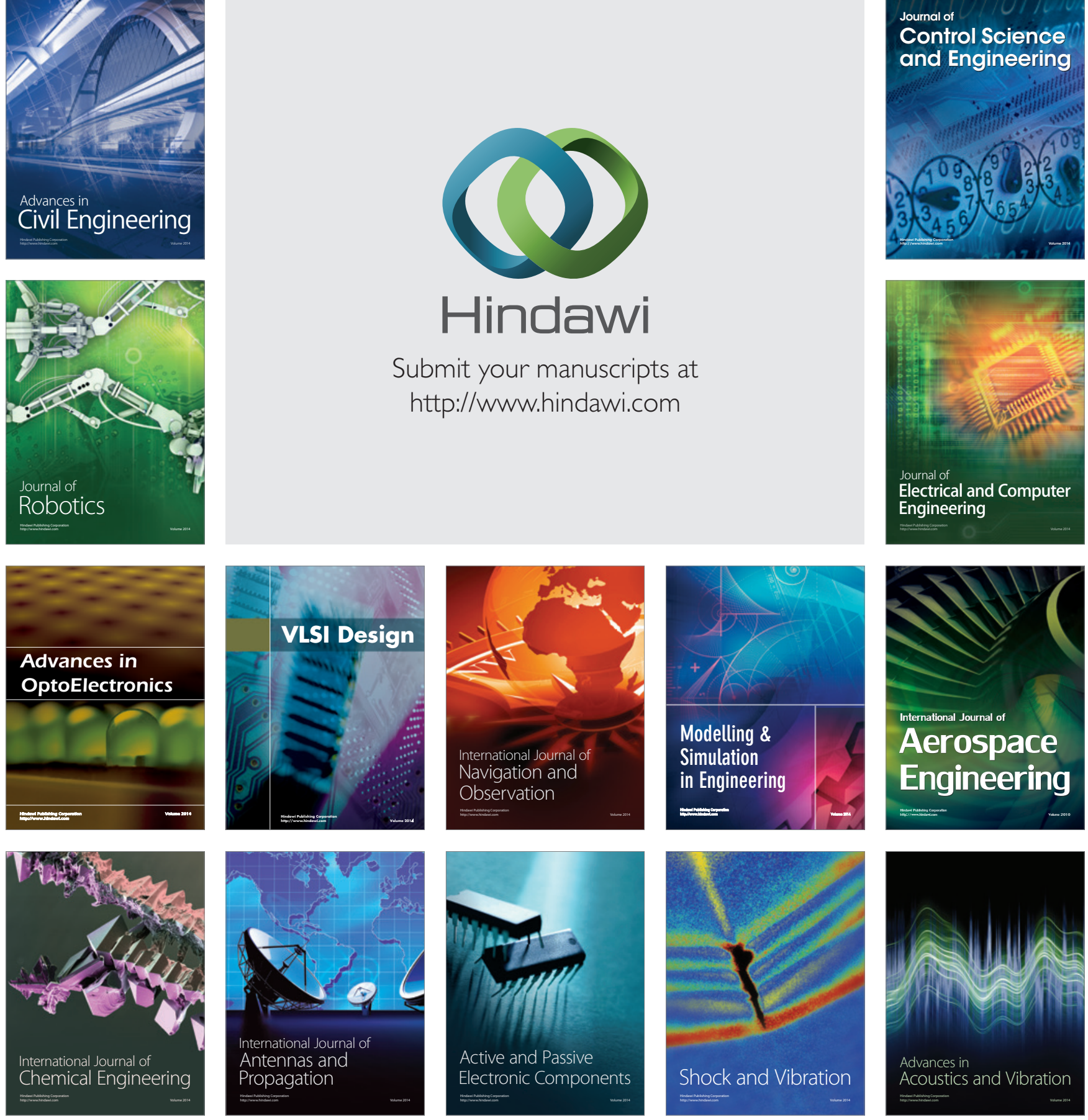